\title{
An LMI Approach to Stability for Linear Time-Varying System with Nonlinear Perturbation on Time Scales
}

\author{
Kanit Mukdasai ${ }^{1}$ and Piyapong Niamsup ${ }^{2,3,4}$ \\ ${ }^{1}$ Department of Mathematics, Faculty of Science, Khon Kaen University, Khon Kaen 40002, Thailand \\ ${ }^{2}$ Department of Mathematics, Faculty of Science, Chiang Mai University, Chiang Mai, 50200, Thailand \\ ${ }^{3}$ Center of Excellence in Mathematics CHE, Si Ayutthaya Road, Bangkok 10400, Thailand \\ ${ }^{4}$ Materials Science Research Center, Faculty of Science, Chiang Mai University, \\ Chiangmai 50200, Thailand
}

Correspondence should be addressed to Kanit Mukdasai, kanit@kku.ac.th

Received 17 December 2010; Revised 3 May 2011; Accepted 19 May 2011

Academic Editor: Martin D. Schechter

Copyright (C) 2011 K. Mukdasai and P. Niamsup. This is an open access article distributed under the Creative Commons Attribution License, which permits unrestricted use, distribution, and reproduction in any medium, provided the original work is properly cited.

We consider Lyapunov stability theory of linear time-varying system and derive sufficient conditions for uniform stability, uniform exponential stability, $\psi$-uniform stability, and $h$-stability for linear time-varying system with nonlinear perturbation on time scales. We construct appropriate Lyapunov functions and derive several stability conditions. Numerical examples are presented to illustrate the effectiveness of the theoretical results.

\section{Introduction}

In the past decades, stability analysis of dynamic systems has become an important topic both theoretically and practically because dynamic systems occur in many areas such as mechanics, physics, and economics. The theory of dynamic equations on time scales was first introduced by Hilger [1] with analysis of measure chains in order to unify continuous and discrete calculus on time scale. The generalized derivative or Hilger derivative $f^{\Delta}(t)$ of a function $f: \mathbb{T} \rightarrow \mathbb{R}$, where $\mathbb{T}$ is a so-called time scale (an arbitrary closed nonempty subset of $\mathbb{R}$ ) becomes the usual derivative when $\mathbb{T}=\mathbb{R}$, namely, $f^{\Delta}(t)=f^{\prime}(t)$. On the other hand, if $\mathbb{T}=\mathbb{Z}$, then $f^{\Delta}(t)$ reduces to the usual forward difference, namely, $f^{\Delta}(t)=\Delta f(t)$. The development of theory on time scale calculus allows one to get some insight into and better understanding of the subtle differences between discrete and continuous systems $[2,3]$. Therefore, the problem of stability analysis for dynamic equations (systems) on time scales 
has been investigated by many researchers, see [1-6], in which most results on stability of dynamic systems are obtained by the method of estimation of general solution of the systems. It seems that there are not many researches concerning with stability of dynamic systems on time scales by using Lyapunov functions on time scales.

There are various types of stability of dynamic systems on time scales such as uniform stability, uniform asymptotic stability [5], $\psi$-uniform stability [6], and $h$-stability [4]. In [5], necessary and sufficient conditions for uniform stability and uniform asymptotic stability for dynamic systems on time scales are obtained. In [4, 6], the method presents in [5] are used to derive sufficient conditions for $\psi$-uniformly stability [6] and $h$-stability [4] for dynamic systems on time scales.

In this paper, we shall develop Lyapunov stability theory for various types of stability for linear time-varying system with nonlinear perturbation on time scales. By using this Lyapunov stability theory, we derive several sufficient conditions for stabilities of dynamic systems on time scales.

\section{Problem Formulation and Preliminaries}

In this section, we introduce some notations, definitions, and preliminary results which will be used throughout the paper. $\mathbb{R}^{+}$denotes the set of all nonnegative real numbers; $\mathbb{R}$ denotes the set of all real numbers; $\mathbb{Z}^{+}$denotes the set of all non-negative integers; $\mathbb{Z}$ denotes the set of all integers; $\mathbb{R}^{n}$ denotes the $n$-dimensional Euclidean space with the usual Euclidean norm $\|\cdot\| ;\|x\|$ denotes the Euclidean vector norm of $x \in \mathbb{R}^{n} ; \mathbb{R}^{n \times r}$ denotes the set of $n \times r$ real matrix; $A^{T}$ denotes the transpose of the matrix $A$; $A$ is symmetric if $A=A^{T}$; $I$ denotes the identity matrix; $\lambda(A)$ denotes the set of all eigenvalues of $A ; \lambda_{\max }(A)=\max \{\operatorname{Re} \lambda: \lambda \in \lambda(A)\}$; $\lambda_{\min }(A)=\min \{\operatorname{Re} \lambda: \lambda \in \lambda(A)\}$.

Definition 2.1. A time scale $\mathbb{T}$ is an arbitrary nonempty closed subset of the real numbers $\mathbb{R}$.

Definition 2.2. The mapping $\sigma, \rho: \mathbb{T} \rightarrow \mathbb{T}$ defined by $\sigma(t)=\inf \{s \in \mathbb{T}: s>t\}$, and $\rho(t)=$ $\sup \{s \in \mathbb{T}: s<t\}$ are called the jump operators.

Definition 2.3. A nonmaximal element $t \in \mathbb{T}$ is said to be right-scattered (rs) if $\sigma(t)>t$ and right-dense (rd) if $\sigma(t)=t$. A nonminimal element $t \in \mathbb{T}$ is called left-scattered (ls) if $\rho(t)<t$ and left-dense (ld) if $\rho(t)=t$.

Definition 2.4. The mapping $\mu: \mathbb{T} \rightarrow \mathbb{R}^{+}$defined by $\mu(t)=\sigma(t)-t$ is called the graininess function.

Definition 2.5. (Delta derivative) assume $f: \mathbb{T} \rightarrow \mathbb{R}$ is a function and let $t \in \mathbb{T}$. Then we define $f^{\Delta}(t)$ to be the number (provided it exists) with the property that given any $\epsilon>0$, there is a neighborhood $U$ of $t$ (i.e., $U=(t-\delta, t+\delta) \cap \mathbb{T}$ for some $\delta>0)$ such that $\mid[f(\sigma(t))-$ $f(s)]-f^{\Delta}(t)[\sigma(t)-s]|\leq \epsilon| \sigma(t)-s \mid$ for all $s \in U$.

The function $f^{\Delta}(t)$ is the delta derivative of $f$ at $t$.

In the case that $\mathbb{T}=\mathbb{R}$, we have $f^{\Delta}(t)=f^{\prime}(t)$. In the case that $\mathbb{T}=\mathbb{Z}$, we have $f^{\Delta}(t)=$ $f(t+1)-f(t)$. 
The following are some useful relationships regarding the delta derivative, see [2].

Theorem 2.6 (see [2]). Assume that $f: \mathbb{T} \rightarrow \mathbb{R}^{n}$ and let $t \in \mathbb{T}$.

(i) If $f$ is differentiable at $t$, then $f$ is continuous at $t$.

(ii) If $f$ is continuous at $t$ and $t$ is right scattered, then $f$ is differentiable at $t$ with

$$
f^{\Delta}(t)=\frac{f(\sigma(t))-f(t)}{\sigma(t)-t}
$$

(iii) If $f$ is differentiable at $t$ and $t$ is right dense, then

$$
f^{\Delta}(t)=\lim _{s \rightarrow t} \frac{f(t)-f(s)}{t-s}
$$

(iv) If $f$ is differentiable at $t$, then

$$
f(\sigma(t))=f(t)+\mu(t) f^{\Delta}(t) .
$$

Theorem 2.7 (see [2]). Assume that $f, g: \mathbb{T} \rightarrow \mathbb{R}^{n}$ and let $t \in \mathbb{T}$.

(i) The sum $f, g: \mathbb{T} \rightarrow \mathbb{R}^{n}$ are differentiable at $t$ with

$$
(f+g)^{\Delta}(t)=(f)^{\Delta}(t)+(g)^{\Delta}(t) .
$$

(ii) For any constant $\alpha, \alpha f: \mathbb{T} \rightarrow \mathbb{R}^{n}$ is differentiable at $t$ with

$$
(\alpha f)^{\Delta}(t)=\alpha f^{\Delta}(t)
$$

(iii) The product $f g: \mathbb{T} \rightarrow \mathbb{R}^{n}$ is differentiable at $t$ with

$$
(f g)^{\Delta}(t)=f^{\Delta}(t) g(t)+f(\sigma(t)) g^{\Delta}(t)=f(t) g^{\Delta}(t)+f^{\Delta}(t) g(\sigma(t)) .
$$

Definition 2.8. The function $f: \mathbb{T} \rightarrow \mathbb{R}^{n}$ is said to be rd-continuous (denoted by $f \in$ $\left.\mathcal{C}_{\mathrm{rd}}\left(\mathbb{T}, \mathbb{R}^{n}\right)\right)$ if the following conditions hold. 
(i) $f$ is continuous at every right-dense point $t \in \mathbb{T}$.

(ii) $\lim _{s \rightarrow t^{-}} f(s)$ exists and is finite at every ld-point $t \in \mathbb{T}$.

Definition 2.9. Let $f \in \mathcal{C}_{\text {rd }}\left(\mathbb{T}, \mathbb{R}^{n}\right)$. Then $g: \mathbb{T} \rightarrow \mathbb{R}^{n}$ is called the antiderivative of $f$ on $\mathbb{T}$ if it is differentiable on $\mathbb{T}$ and satisfies $g^{\Delta}(t)=f(t)$ for $t \in \mathbb{T}$. In this case, we define

$$
\int_{a}^{t} f(s) \Delta s=g(t)-g(a), \quad a \leq t \in \mathbb{T} .
$$

Consider the linear time-varying system with nonlinear perturbation on time scales $(\mathbb{T})$ of the form

$$
x^{\Delta}(t)=A(t) x(t)+f(t, x(t)), \quad t \in \mathbb{T}
$$

where $x(t) \in \mathbb{R}^{n}, A: \mathbb{T} \rightarrow \mathbb{R}^{n \times n}$ is an $n \times n$ matrix-valued function and $f: \mathbb{T} \times \mathbb{R}^{n} \rightarrow \mathbb{R}^{n}$ is rd-continuous in the first argument with $f(t, 0)=0$. The uncertain perturbation is known to satisfy a bound of the form

$$
\|f(t, x(t))\| \leq \gamma\|x(t)\|
$$

or equivalently, the perturbation is conically bounded. The solution of (2.8) through $\left(t_{0}, x\left(t_{0}\right)\right)$ satisfies the variation of constants formula

$$
x(t)=\Phi_{A}\left(t, t_{0}\right) x\left(t_{0}\right)+\int_{t_{0}}^{t} \Phi_{A}(t, \sigma(s)) f(s, x(s)) \Delta s, \quad t \geq t_{0} .
$$

When $f(t, x(t))=0,(2.8)$ becomes the linear time-varying system

$$
x^{\Delta}(t)=A(t) x(t), \quad x\left(t_{0}\right)=x_{0}, \quad t_{0} \in \mathbb{T} .
$$

For the case when $f(t, x(t))=B(t) x(t), B(t) \in \mathbb{R}^{n \times n}$, (2.8) becomes the linear time-varying system

$$
x^{\Delta}(t)=[A(t)+B(t)] x(t), \quad x\left(t_{0}\right)=x_{0}, \quad t_{0} \in \mathbb{T} .
$$

The norm of $n \times n$ matrix $A$ is defined as

$$
\|A\|=\max _{\|x\|=1}\|A x\|
$$

The Euclidean norm of $n \times 1$ vector $x(t)$ is defined by

$$
\|x(t)\|=\sqrt{x^{T}(t) x(t)} .
$$


Definition 2.10. A function $\phi:[0, r] \rightarrow[0,+\infty)$ is of class $\mathcal{K}$ if it is well-defined, continuous, and strictly increasing on $[0, r]$ with $\phi(0)=0$.

Definition 2.11. Assume $g: \mathbb{T} \rightarrow \mathbb{R}$. Define and denote $g \in \mathcal{C}_{\mathrm{rd}}(\mathbb{T} ; \mathbb{R})$ as right-dense continuous (rd-continuous) if $g$ is continuous at every right-dense point $t \in \mathbb{T}$ and $\lim _{s \rightarrow t^{-}} g(s)$ exists, and is finite, at every left-dense point $t \in \mathbb{T}$. Now define the so-called set of regressive functions, $\mathcal{R}$, by

$$
\mathcal{R}=\left\{p: \mathbb{T} \rightarrow \mathbb{R} \mid p \in \mathcal{C}_{\mathrm{rd}}(\mathbb{T} ; \mathbb{R}), 1+p(t) \mu(t) \neq 0, t \in \mathbb{T}\right\},
$$

and define the set of positively regressive functions by

$$
\mathcal{R}^{+}=\{p \in \mathbb{R} \mid 1+p(t) \mu(t)>0, t \in \mathbb{T}\} .
$$

Definition 2.12. The zero solution of system (2.8) is called uniformly stable if there exists a finite constant $\gamma>0$ such that

$$
\left\|x\left(t, x_{0}, t_{0}\right)\right\| \leq \gamma\left\|x_{0}\right\|
$$

for all $t \in \mathbb{T}, t \geq t_{0}$.

Definition 2.13. The zero solution of system (2.8) is called uniformly exponentially stable if there exist finite constants $\gamma, \lambda>0$ with $-\lambda \in \mathcal{R}^{+}$such that

$$
\left\|x\left(t, x_{0}, t_{0}\right)\right\| \leq \gamma\left\|x_{0}\right\| e_{-\lambda}\left(t, t_{0}\right)
$$

for all $t \in \mathbb{T}, t \geq t_{0}$.

Definition 2.14. The zero solution of system (2.8) is called $\psi$-uniformly stable if there exists a finite constant $\gamma>0$ such that for any $t_{0}$ and $x\left(t_{0}\right)$, the corresponding solution satisfies

$$
\left\|\psi(t) x\left(t, x_{0}, t_{0}\right)\right\| \leq \gamma\left\|\psi\left(t_{0}\right) x_{0}\right\|
$$

for all $t \in \mathbb{T}, t \geq t_{0}$.

Definition 2.15. System (2.8) is called an $h$-system if there exist a positive function $h: \mathbb{T} \rightarrow \mathbb{R}$, a constant $c \geq 1$ and $\delta>0$ such that

$$
\left\|x\left(t, x_{0}, t_{0}\right)\right\| \leq c\left\|x_{0}\right\| h(t) h\left(t_{0}\right)^{-1}, \quad t \geq t_{0}
$$

if $\left\|x_{0}\right\|<\delta$ (here $\left.h(t)^{-1}=1 / h(t)\right)$. If $h$ is bounded, then (2.8) is said to be $h$-stable.

Definition 2.16. A continuous function $P: \mathbb{T} \rightarrow \mathbb{R}$ with $P(0)=0$ is called positive definite (negative definite) on $\mathbb{T}$ if there exists a function $\phi \in \mathcal{K}$ such that $\phi(t) \leq P(t)(\phi(t) \leq-P(t))$ for all $t \in \mathbb{T}$. 
Definition 2.17. A continuous function $P: \mathbb{T} \rightarrow \mathbb{R}$ with $P(0)=0$ is called positive semidefinite (negative semi-definite) on $\mathbb{T}$ if $P(t) \geq 0(P(t) \leq 0)$ for all $t \in \mathbb{T}$.

Definition 2.18. A continuous function $P: \mathbb{T} \times \mathbb{R}^{n} \rightarrow \mathbb{R}$ with $P(t, 0)=0$ is called positive definite (negative definite) on $\mathbb{T} \times \mathbb{R}^{n}$ if there exists a function $\phi \in \mathcal{K}$ such that $\phi(\|x\|) \leq P(t, x)$ $(\phi(\|x\|) \leq-P(t, x))$ for all $t \in \mathbb{T}$ and $x \in \mathbb{R}^{n}$.

Definition 2.19. A continuous function $P: \mathbb{T} \times \mathbb{R}^{n} \rightarrow \mathbb{R}$ with $P(t, 0)=0$ is called positive semi-definite (negative semi-definite) on $\mathbb{T} \times \mathbb{R}^{n}$ if $0 \leq P(t, x)(0 \geq P(t, x))$ for all $t \in \mathbb{T}$ and $x \in \mathbb{R}^{n}$.

Lemma 2.20 ([7], Completing the square). assume that $S \in M^{n \times n}$ is a symmetric positive definite matrix. Then for every $Q \in M^{n \times n}$, we obtain

$$
2 x^{T} Q y-y^{T} S y \leq x^{T} Q S^{-1} Q^{T} x, \quad \forall x, y \in R^{n} .
$$

\section{Main Results}

In this section, we first introduce Lyapunov stability theory of various types stability for linear time varying system with nonlinear perturbation on time scales. Then, we use this Lyapunov stability theory to obtain sufficient conditions for various types of stabilities of this system.

\subsection{Lyapunov Stability Theory}

Theorem 3.1. If there exist a continuously differentiable positive definite function $V(t, x(t)) \in$ $\mathcal{C}_{\mathrm{rd}}^{1}\left(\mathbb{T} \times \mathbb{R}^{n}, \mathbb{R}^{+}\right)$, and $a, b \in \mathbb{R}^{+}$such that

(i) $V^{\Delta}(t, x(t)) \leq 0$,

(ii) $a\|x(t)\|^{2} \leq V(t, x(t)) \leq b\|x(t)\|^{2}$,

then the zero solution of system (2.8) is $\psi$-uniformly stable if there exists $\psi(t) \in \mathcal{C}_{\mathrm{rd}}^{1}\left(\mathbb{T}, \mathbb{R}_{+}\right)$satisfying $\psi^{\Delta}(t) \leq 0$.

Proof. For $t_{0} \in \mathbb{T}$, we let $x\left(t_{0}\right)=x_{0}$. Then, by (i), we have

$$
\begin{gathered}
\int_{t_{0}}^{t} V^{\Delta}(s, x(s)) \Delta s=V(t, x(t))-V\left(t_{0}, x\left(t_{0}\right)\right) \leq 0, \\
\int_{t_{0}}^{t} \psi^{\Delta}(s) \Delta s=\psi(t)-\psi\left(t_{0}\right) \leq 0 .
\end{gathered}
$$

We obtain $V(t, x(t)) \leq V\left(t_{0}, x\left(t_{0}\right)\right)$ and $\psi(t) \leq \psi\left(t_{0}\right)$ for all $t \in \mathbb{T}, t \geq t_{0}$. By (ii), we get the estimation as follows:

$$
a\|\psi(t)\|^{2}\|x(t)\|^{2} \leq\|\psi(t)\|^{2} V(t, x(t)) \leq\left\|\psi\left(t_{0}\right)\right\|^{2} V\left(t_{0}, x\left(t_{0}\right)\right) \leq b\left\|\psi\left(t_{0}\right)\right\|^{2}\left\|x\left(t_{0}\right)\right\|^{2} .
$$

We conclude that $\|\psi(t) x(t)\| \leq \gamma\left\|\psi\left(t_{0}\right) x\left(t_{0}\right)\right\|$ where $\gamma=\sqrt{b / a}>0$. Therefore, the zero solution of system (2.8) is $\psi$-uniformly stable. The proof of the theorem is complete. 
Corollary 3.2. If there exist a continuously differentiable positive definite function $V(t, x(t)) \in$ $\mathcal{C}_{\text {rd }}^{1}\left(\mathbb{T} \times \mathbb{R}^{n}, \mathbb{R}^{+}\right)$and $a, b \in \mathbb{R}^{+}$such that

(i) $V^{\Delta}(t, x(t)) \leq 0$,

(ii) $a\|x(t)\|^{2} \leq V(t, x(t)) \leq b\|x(t)\|^{2}$,

then the zero solution of system (2.8) is uniformly stable.

Theorem 3.3. If there exist a continuously differentiable positive definite function $V(t, x(t)) \in$ $\mathcal{C}_{\mathrm{rd}}^{1}\left(\mathbb{T} \times \mathbb{R}^{n}, \mathbb{R}^{+}\right)$and $a, b, \epsilon \in \mathbb{R}^{+}$with $-\epsilon / b \in \mathcal{R}^{+}$satisfying

(i) $V^{\Delta}(t, x(t)) \leq-\epsilon\|x(t)\|^{2}$,

(ii) $a\|x(t)\|^{2} \leq V(t, x(t)) \leq b\|x(t)\|^{2}$,

then the zero solution of system (2.8) is uniformly exponentially stable.

Proof. For $t_{0} \in \mathbb{T}$, we let $x\left(t_{0}\right)=x_{0}$. We obtain, by (i) and (ii), that for all $t \geq t_{0}$,

$$
V^{\Delta}(t, x(t)) \leq-\epsilon\|x(t)\|^{2} \leq-\frac{\epsilon}{b} V(t, x(t))
$$

Since $-\epsilon / b \in \mathcal{R}^{+}$, it follows from Gronwall's inequality for time scales [2] and (ii) that

$$
a\|x(t)\|^{2} \leq V(t, x(t)) \leq V\left(t_{0}, x\left(t_{0}\right)\right) e_{-\epsilon / b}\left(t, t_{0}\right) \leq b\left\|x\left(t_{0}\right)\right\|^{2} e_{-\epsilon / b}\left(t, t_{0}\right) .
$$

Hence, we get

$$
\|x(t)\| \leq \gamma\left\|x\left(t_{0}\right)\right\|\left[e_{-\epsilon / b}\left(t, t_{0}\right)\right]^{1 / 2},
$$

where $r=\sqrt{b / a}$ for all $t \geq t_{0}$. Therefore, the zero solution of system (2.8) is uniformly exponentially stable. The proof of the theorem is complete.

Theorem 3.4. If there exist a continuously differentiable positive definite function $V(t, x(t)) \in$ $\mathcal{C}_{\mathrm{rd}}^{1}\left(\mathbb{T} \times \mathbb{R}^{n}, \mathbb{R}^{+}\right)$, a bounded positive differentiable function $h: \mathbb{T} \rightarrow \mathbb{R}$ and $a, b \in \mathbb{R}^{+}$such that

(i)

$$
V^{\Delta}(t, x(t)) \leq \gamma h^{\Delta}(t) / h(t)\|x(t)\|^{2}, \quad \gamma= \begin{cases}a, & h^{\Delta}(t) \geq 0 \\ b, & h^{\Delta}(t)<0\end{cases}
$$

(ii) $a\|x(t)\|^{2} \leq V(t, x(t)) \leq b\|x(t)\|^{2}$,

then the zero solution of system (2.8) is h-stable. 
Proof. Let $t_{0} \in \mathbb{T}, x\left(t_{0}\right)=x_{0}$ and $x\left(t, t_{0}, x_{0}\right)=x(t)$ be any solution of system (2.8). By (i), we have

$$
\begin{aligned}
V^{\Delta}(t, x(t)) & \leq \gamma \frac{h^{\Delta}(t)}{h(t)}\|x(t)\|^{2} \\
& \leq \begin{cases}\frac{\gamma}{a} \frac{h^{\Delta}(t)}{h(t)} V(t, x(t)), & h^{\Delta}(t) \geq 0 \\
\frac{\gamma}{b} \frac{h^{\Delta}(t)}{h(t)} V(t, x(t)), & h^{\Delta}(t)<0,\end{cases} \\
& \leq \frac{h^{\Delta}(t)}{h(t)} V(t, x(t)) .
\end{aligned}
$$

From Gronwall's inequality for time scales [2], (ii) and Lemma 2.15 [4], we obtain

$$
\begin{aligned}
a\|x(t)\|^{2} & \leq V(t, x(t)) \leq V\left(t_{0}, x\left(t_{0}\right)\right) e_{h^{\Delta}(t) / h(t)}\left(t, t_{0}\right) \leq b\left\|x\left(t_{0}\right)\right\|^{2} e_{h^{\Delta}(t) / h(t)}\left(t, t_{0}\right), \\
& \leq b\left\|x\left(t_{0}\right)\right\|^{2} \frac{h(t)}{h\left(t_{0}\right)} .
\end{aligned}
$$

Thus,

$$
\|x(t)\| \leq \gamma\left\|x\left(t_{0}\right)\right\| H(t) H\left(t_{0}\right)^{-1}, \quad t \geq t_{0}
$$

where $\gamma=\sqrt{b / a}$ and $H(t)=\sqrt{h(t)}$. Therefore, zero solution of (2.8) is $h$-stable.

\subsection{Stability Conditions}

We introduce the following notation for later use:

$$
\begin{aligned}
Z(t):= & P^{\Delta}(t)+A^{T}(t) P(t)+P(t) A(t)+\mu(t) P^{\Delta}(t) A(t)+\mu(t) A^{T}(t) P^{\Delta}(t)+\epsilon_{1} P(t) P(t) \\
& +\mu(t) A^{T}(t) P(t) A(t)+\mu^{2}(t) A^{T}(t) P^{\Delta}(t) A(t)+\epsilon_{2} P^{\Delta}(t) P^{\Delta}(t)+\epsilon_{2}^{-1} \gamma^{2} \mu(t)^{2} I \\
& +\epsilon_{3} A^{T}(t) P(t) P(t) A(t)+\epsilon_{3}^{-1} \gamma^{2} \mu(t)^{2} I+\epsilon_{4} A^{T}(t) P^{\Delta}(t) P^{\Delta}(t) A(t)+\epsilon_{4}^{-1} \gamma^{2} \mu(t)^{4} I \\
& +\epsilon_{1}^{-1} \gamma^{2} I+\eta_{2} \gamma^{2} \mu(t) I+\rho_{2} \gamma^{2} \mu(t)^{2} I .
\end{aligned}
$$

Theorem 3.5. The system (2.11) is uniformly stable if there exist a positive definite symmetric matrix function $P(t) \in \mathcal{C}_{\mathrm{rd}}^{1}\left(\mathbb{T}, \mathbb{R}^{n \times n}\right)$ and $\eta, \rho \in \mathbb{R}^{+}$such that

(i) $\eta I \leq P(t) \leq \rho I$,

(ii) $A^{T}(t) P(t)+\left(I+\mu(t) A^{T}(t)\right)\left(P^{\Delta}(t)+P(t) A(t)+\mu(t) P^{\Delta}(t) A(t)\right) \leq 0$. 
Remark 3.6. We can prove Theorem 3.5 (see Theorem 3.1 in [5] DaCunha) by using the same approach as in Theorem 3.1 by choosing $V(t, x(t))=x^{T}(t) P(t) x(t)$. In this case, we obtain

$$
V^{\Delta}(t)=x^{T}(t)\left[A^{T}(t) P(t)+\left(I+\mu(t) A^{T}(t)\right)\left(P^{\Delta}(t)+P(t) A(t)+\mu(t) P^{\Delta}(t) A(t)\right)\right] x(t) .
$$

Theorem 3.7. The system (2.8) is uniformly stable if there exist a positive definite symmetric matrix function $P(t) \in \mathcal{C}_{\mathrm{rd}}^{1}\left(\mathbb{T}, \mathbb{R}^{n \times n}\right)$ and $\eta_{1}, \eta_{2}, \gamma, \epsilon_{1}, \epsilon_{2}, \epsilon_{3}, \epsilon_{4} \in \mathbb{R}^{+}, \rho_{1}, \rho_{2} \in \mathbb{R}$ such that

(i) $\eta_{1} I \leq P(t) \leq \eta_{2} I$,

(ii) $\rho_{1} I \leq P^{\Delta}(t) \leq \rho_{2} I$,

(iii) $Z(t) \leq 0$.

Proof. We consider the following Lyapunov function for system (2.8).

$$
V(t, x(t))=x^{T}(t) P(t) x(t) .
$$

By (i), it is easy to see that

$$
\eta_{1}\|x(t)\|^{2} \leq V(t, x(t))=x^{T}(t) P(t) x(t) \leq \eta_{2}\|x(t)\|^{2} .
$$

The delta derivative of $V$ along the trajectories of system (2.8) is given by

$$
\begin{aligned}
V^{\Delta}(t)= & {\left[x^{T}(t) P(t)\right]^{\Delta} x(t)+x^{T}(\sigma(t)) P(\sigma(t)) x^{\Delta}(t) } \\
= & x^{T}(t)^{\Delta} P(t) x(t)+x^{T}(\sigma(t)) P^{\Delta}(t) x(t)+x^{T}(\sigma(t)) P(\sigma(t)) x^{\Delta}(t) \\
= & {\left[x^{T}(t) A^{T}(t)+f^{T}(t, x)\right] P(t) x(t)+\left[x^{T}(t)+\mu(t)\left(x^{T}(t) A^{T}(t)+f^{T}(t, x)\right)\right] } \\
& \times\left[P^{\Delta}(t) x(t)+P(t)(A(t) x(t)+f(t, x))+\mu(t) P^{\Delta}(t)(A(t) x(t)+f(t, x))\right] \\
= & x^{T}(t) P^{\Delta}(t) x(t)+x^{T}(t) A^{T}(t) P(t) x(t)+x^{T}(t) P(t) A(t) x(t) \\
& +\mu(t) x^{T}(t) P^{\Delta}(t) A(t) x(t) \\
& +\mu(t) x^{T}(t) A^{T}(t) P^{\Delta}(t) x(t)+\mu(t) x^{T}(t) A^{T}(t) P(t) A(t) x(t) \\
& +\mu(t)^{2} x^{T}(t) A^{T}(t) P^{\Delta}(t) A(t) x(t) \\
& +\mu(t) f^{T}(t, x) P(t) f(t, x)+\mu(t)^{2} f^{T}(t, x) P^{\Delta}(t) f(t, x)+f^{T}(t, x) P(t) x(t) \\
& +\mu(t) f^{T}(t, x) P^{\Delta}(t) x(t)+\mu(t) f^{T}(t, x) P(t) A(t) x(t) \\
& +\mu(t)^{2} f^{T}(t, x) P^{\Delta}(t) A(t) x(t) \\
& +x^{T}(t) P(t) f(t, x)+\mu(t) x^{T}(t) P^{\Delta}(t) f(t, x)+\mu(t) x^{T}(t) A^{T}(t) P(t) f(t, x) \\
& +\mu(t)^{2} x^{T}(t) A^{T}(t) P^{\Delta}(t) f(t, x) .
\end{aligned}
$$


By (i), (ii), and Lemma 2.20, we have the following estimate:

$$
\begin{gathered}
x^{T}(t) P(t) x(t) \leq \eta_{2} x^{T}(t) x(t), \\
x^{T}(t) P^{\Delta}(t) x(t) \leq \rho_{2} x^{T}(t) x(t), \\
2 x^{T}(t) P(t) f(t, x) \leq \epsilon_{1} x^{T}(t) P(t) P(t) x(t)+\epsilon_{1}^{-1} f^{T}(t, x) f(t, x), \\
2 \mu(t) x^{T}(t) P^{\Delta}(t) f(t, x) \leq \epsilon_{2} x^{T}(t) P^{\Delta}(t) P^{\Delta}(t) x(t)+\epsilon_{2}^{-1} \mu(t)^{2} f^{T}(t, x) f(t, x), \\
2 \mu(t) x^{T}(t) A^{T}(t) P(t) f(t, x) \leq \epsilon_{3} x^{T}(t) A^{T}(t) P(t) P(t) A(t) x(t)+\epsilon_{3}^{-1} \mu(t)^{2} f^{T}(t, x) f(t, x), \\
2 \mu(t)^{2} x^{T}(t) A^{T}(t) P^{\Delta}(t) f(t, x) \leq \epsilon_{4} x^{T}(t) A^{T}(t) P^{\Delta}(t) P^{\Delta}(t) A(t) x(t)+\epsilon_{4}^{-1} \mu(t)^{4} f^{T}(t, x) f(t, x) .
\end{gathered}
$$

From the above inequalities and $\|f(t, x)\| \leq \gamma\|x(t)\|$, we obtain

$$
\begin{aligned}
V^{\Delta}(t) \leq & x^{T}(t) P^{\Delta}(t) x(t)+x^{T}(t) A^{T}(t) P(t) x(t)+x^{T}(t) P(t) A(t) x(t) \\
& +\mu(t) x^{T}(t) P^{\Delta}(t) A(t) x(t) \\
& +\mu(t) x^{T}(t) A^{T}(t) P^{\Delta}(t) x(t)+\mu(t) x^{T}(t) A^{T}(t) P(t) A(t) x(t) \\
& +\mu(t)^{2} x^{T}(t) A^{T}(t) P^{\Delta}(t) A(t) x(t) \\
& +\epsilon_{1} x^{T}(t) P(t) P(t) x(t)+\epsilon_{1}^{-1} \gamma^{2}\|x(t)\|^{2}+\epsilon_{2} x^{T}(t) P^{\Delta}(t) P^{\Delta}(t) x(t) \\
& +\epsilon_{2}^{-1} \gamma^{2} \mu(t)^{2}\|x(t)\|^{2} \\
& +\epsilon_{3} x^{T}(t) A^{T}(t) P(t) P(t) A(t) x(t)+\epsilon_{3}^{-1} \gamma^{2} \mu(t)^{2}\|x(t)\|^{2}+\epsilon_{4}^{-1} \gamma^{2} \mu(t)^{4}\|x(t)\|^{2} \\
& +\epsilon_{4} x^{T}(t) A^{T}(t) P^{\Delta}(t) P^{\Delta}(t) A(t) x(t)+\eta_{2} \gamma^{2} \mu(t)\|x(t)\|^{2}+\rho_{2} \gamma^{2} \mu(t)^{2}\|x(t)\|^{2} \\
= & x^{T}(t) Z(t) x(t) .
\end{aligned}
$$

By (iii), we conclude that $V^{\Delta}(t) \leq 0$. Therefore, the zero solution of (2.8) is uniformly stable by Corollary 3.2.

Example 3.8. We consider the time-varying dynamic system of the form

$$
\begin{gathered}
x^{\Delta}(t)=A(t) x(t)+f(t, x(t)), \\
A(t)=\left[\begin{array}{cc}
-a(t) & -1 \\
1 & -a(t)
\end{array}\right], \quad f(t, x(t))=\left[\begin{array}{c}
-0.125 \sin (t)\left[x_{2}(t)\right] \\
0.125 \cos (t)\left[x_{1}(t)\right]
\end{array}\right], \quad\|f(t, x(t))\| \leq 0.125\|x(t)\|,
\end{gathered}
$$

where $a(t)=-e_{\ominus 8}(t, 0)+1$ and $f(t, x(t))$ are rd-continuous in the first argument with $f(t, 0)=0$ for all $t \in \mathbb{T}$. Let $\gamma=1 / 8, \epsilon_{1}=1, \epsilon_{2}=1 / 16, \epsilon_{3}=1 / 2, \epsilon_{4}=1 / 16, \eta_{1}=1 / 8, \eta_{2}=1 / 4, \rho_{1}=-1$, 
and $\rho_{2}=0$. By assuming that $0 \leq \mu(t) \leq 0.25$ for all $t \in \mathbb{T}$, we can find solution $P(t)$ satisfying conditions (i)-(iii) of Theorem 3.7 as $P(t)=\left[\begin{array}{cc}(1 / 8) e_{\ominus 8}(t, 0)+(1 / 8) & 0 \\ 0 & (1 / 8) e_{\ominus 8}(t, 0)+(1 / 8)\end{array}\right]$. Observe that,

$$
P^{\Delta}(t)=\left[\begin{array}{cc}
-e_{\ominus 8}(t, 0) & 0 \\
0 & -e_{\ominus 8}(t, 0)
\end{array}\right]
$$

Therefore, by Theorem 3.7, the system (3.17) is uniformly stable.

Theorem 3.9. The system (2.8) is uniformly exponentially stable if there exist positive definite symmetric matrix function $P(t) \in \mathcal{C}_{\mathrm{rd}}^{1}\left(\mathbb{T}, \mathbb{R}^{n \times n}\right)$ and $\eta_{1}, \eta_{2}, \gamma, \epsilon_{1}, \epsilon_{2}, \epsilon_{3}, \epsilon_{4}, \epsilon_{5} \in \mathbb{R}^{+}, \rho_{1}, \rho_{2} \in \mathbb{R}$ such that

(i) $\eta_{1} I \leq P(t) \leq \eta_{2} I$,

(ii) $\rho_{1} I \leq P^{\Delta}(t) \leq \rho_{2} I$,

(iii) $Z(t) \leq-\epsilon_{5} I$.

Proof. Consider a Lyapunov function for system (2.8) of the form

$$
V(t, x(t))=x^{T}(t) P(t) x(t)
$$

It is easy to see that (i) yields

$$
\eta_{1}\|x(t)\|^{2} \leq V(t, x(t))=x^{T}(t) P(t) x(t) \leq \eta_{2}\|x(t)\|^{2} .
$$

The delta derivative of $V$ along the trajectories of system (2.8) is given by

$$
\begin{aligned}
V^{\Delta}(t)= & {\left[x^{T}(t) P(t)\right]^{\Delta} x(t)+x^{T}(\sigma(t)) P(\sigma(t)) x^{\Delta}(t) } \\
= & {\left[x^{T}(t) A^{T}(t)+f^{T}(t, x)\right] P(t) x(t)+\left[x^{T}(t)+\mu(t)\left(x^{T}(t) A^{T}(t)+f^{T}(t, x)\right)\right] } \\
& \times\left[P^{\Delta}(t) x(t)+P(t)(A(t) x(t)+f(t, x))+\mu(t) P^{\Delta}(t)(A(t) x(t)+f(t, x))\right] .
\end{aligned}
$$

From Theorem 3.7, we obtain

$$
\begin{aligned}
V^{\Delta}(t) \leq & x^{T}(t) P^{\Delta}(t) x(t)+x^{T}(t) A^{T}(t) P(t) x(t)+x^{T}(t) P(t) A(t) x(t)+\mu(t) x^{T}(t) P^{\Delta}(t) A(t) x(t) \\
& +\mu(t) x^{T}(t) A(t) P^{\Delta}(t) x(t)+\mu(t) x^{T}(t) A(t) P(t) A(t) x(t)+\mu(t)^{2} x^{T}(t) A(t) P^{\Delta}(t) A(t) x(t) \\
& +\epsilon_{1} x^{T}(t) P(t) P(t) x(t)+\epsilon_{1}^{-1} \gamma^{2}\|x(t)\|^{2}+\epsilon_{2} x^{T}(t) P^{\Delta}(t) P^{\Delta}(t) x(t)+\epsilon_{2}^{-1} \gamma^{2} \mu(t)^{2}\|x(t)\|^{2} \\
& +\epsilon_{3} x^{T}(t) A^{T}(t) P(t) P(t) A(t) x(t)+\epsilon_{3}^{-1} \gamma^{2} \mu(t)^{2}\|x(t)\|^{2}+\epsilon_{4}^{-1} \gamma^{2} \mu(t)^{4}\|x(t)\|^{2} \\
& +\epsilon_{4} x^{T}(t) A^{T}(t) P^{\Delta}(t) P^{\Delta}(t) A(t) x(t)+\eta_{2} \gamma^{2} \mu(t)\|x(t)\|^{2}+\rho_{2} \gamma^{2} \mu(t)^{2}\|x(t)\|^{2} .
\end{aligned}
$$


By (iii), we conclude that $V^{\Delta}(t) \leq-\epsilon_{5}\|x(t)\|^{2}$. By Theorem 3.3, the zero solution of (2.8) is uniformly exponentially stable.

Example 3.10. We consider the linear time-varying system with nonlinear perturbation of the form

$$
x^{\Delta}(t)=A(t) x(t)+f(t, x(t))
$$

where

$$
A(t)=\left[\begin{array}{cc}
-a(t) & -1 \\
1 & -a(t)
\end{array}\right], \quad f(t, x(t))=\left[\begin{array}{c}
-0.125 \cos (t)\left[x_{2}(t)\right] \\
0.125 \sin (t)\left[x_{1}(t)\right]
\end{array}\right] .
$$

$a(t)=e_{\ominus 8}(t, 0)+1$ and $f(t, x(t))$ are rd-continuous in the first argument with $f(t, 0)=0$ for all $t \in \mathbb{T}$. Then, $1 \leq a(t) \leq 2$ and $\|f(t, x(t))\| \leq 0.125\|x(t)\|$ for all $t \in \mathbb{T}$. Let $\gamma=1 / 8, \epsilon_{1}=1, \epsilon_{2}=$ $1 / 16, \epsilon_{3}=1 / 2, \epsilon_{4}=\epsilon_{5}=1 / 16, \eta_{1}=1 / 8, \eta_{2}=1 / 4, \rho_{1}=-1$, and $\rho_{2}=0$. By assuming that $0 \leq \mu(t) \leq 0.25$, for all $t \in \mathbb{T}$, we can find a solution $P(t)$ satisfying (i)-(iii) of Theorem 3.9 as

$$
P(t)=\left[\begin{array}{ccc}
\frac{1}{8} e_{\ominus 8}(t, 0)+\frac{1}{8} & 0 \\
0 & \frac{1}{8} e_{\ominus 8}(t, 0)+\frac{1}{8}
\end{array}\right] .
$$

Therefore, by Theorem 3.9, the system (3.24) is uniformly exponentially stable.

Theorem 3.11. The system (2.8) is $\psi$-uniformly stable if there exist positive definite symmetric matrix function $P(t) \in \mathcal{C}_{\mathrm{rd}}^{1}\left(\mathbb{T}, \mathbb{R}^{n \times n}\right), \psi(t) \in \mathcal{C}_{\mathrm{rd}}^{1}\left(\mathbb{T}, \mathbb{R}_{+}\right)$, and $\eta_{1}, \eta_{2}, \gamma, \epsilon_{1}, \epsilon_{2}, \epsilon_{3}, \epsilon_{4} \in \mathbb{R}^{+}, \rho_{1}, \rho_{2} \in \mathbb{R}$ such that

(i) $\eta_{1} I \leq P(t) \leq \eta_{2} I$,

(ii) $\rho_{1} I \leq P^{\Delta}(t) \leq \rho_{2} I$,

(iii) $Z(t) \leq 0$,

(iv) $\psi^{\Delta}(t) \leq 0$.

Proof. We consider the following Lyapunov function for system (2.8)

$$
V(t, x(t))=x^{T}(t) P(t) x(t)
$$

By (i), it is easy to see that

$$
\eta_{1}\|x(t)\|^{2} \leq V(t, x(t))=x^{T}(t) P(t) x(t) \leq \eta_{2}\|x(t)\|^{2} .
$$

By the same argument as in the proof of Theorem 3.7, we obtain $V^{\Delta}(t) \leq 0$. By (iv) and Theorem 3.1, the zero solution of (2.8) is $\psi$-uniformly stable. 
Example 3.12. We consider the linear time-varying dynamic system of the form

$$
x^{\Delta}(t)=\left[\begin{array}{cc}
-a(t) & -1 \\
1 & -a(t)
\end{array}\right] x(t)+f(t, x(t)),
$$

$a(t)=|\sin (t)|+1$ and $f(t, x(t))$ are rd-continuous in the first argument with $f(t, 0)=0$ for all $t \in \mathbb{T}$. We let $\psi(t)=-t$ and

$$
f(t, x(t))=\left[\begin{array}{c}
0.125 \sin (t)\left[x_{1}(t)\right] \\
-0.125 \cos (t)\left[x_{2}(t)\right]
\end{array}\right] .
$$

Then $\psi^{\Delta}(t)=-1 \leq 0$ and $\|f(t, x(t))\| \leq 0.125\|x(t)\|$. Let $\gamma=1 / 8, \epsilon_{1}=1, \epsilon_{2}=1 / 16, \epsilon_{3}=$ $1 / 2, \epsilon_{4}=1 / 16, \eta_{1}=1 / 8, \eta_{2}=1 / 4, \rho_{1}=-1$, and $\rho_{2}=0$. We can find a solution $P(t)$ satisfying (i)-(iv) of Theorem 3.11 as

$$
P(t)=\left[\begin{array}{cc}
\frac{1}{8} e_{\ominus 8}(t, 0)+\frac{1}{8} & 0 \\
0 & \frac{1}{8} e_{\ominus 8}(t, 0)+\frac{1}{8}
\end{array}\right]
$$

$\psi^{\Delta}(t)=-1 \leq 0$.

Therefore, by Theorem 3.11, the system (3.29) is $\psi$-uniformly stable.

Theorem 3.13. The system (2.8) is $h$-stable if there exist a positive definite symmetric matrix function $P(t) \in \mathcal{C}_{\mathrm{rd}}^{1}\left(\mathbb{T}, \mathbb{R}^{n \times n}\right)$, a bounded positive differentiable function $h: \mathbb{T} \rightarrow \mathbb{R}$, and $\eta_{1}, \eta_{2}$, $\gamma, \epsilon_{1}, \epsilon_{2}, \epsilon_{3}, \epsilon_{4} \in \mathbb{R}^{+}, \rho_{1}, \rho_{2} \in \mathbb{R}$ satisfying

(i) $\eta_{1} I \leq P(t) \leq \eta_{2} I$,

(ii) $\rho_{1} I \leq P^{\Delta}(t) \leq \rho_{2} I$,

(iii)

$$
Z(t) \leq \gamma_{1} \frac{h^{\Delta}(t)}{h(t)} I, \quad r_{1}= \begin{cases}\eta_{1}, & h^{\Delta}(t) \geq 0 \\ \eta_{2}, & h^{\Delta}(t)<0 .\end{cases}
$$

Proof. Let $t_{0} \in \mathbb{T}, x\left(t_{0}\right)=x_{0}$ and $x\left(t, t_{0}, x_{0}\right)=x(t)$ be any solution of system (2.8). We consider a Lyapunov function for system (2.8) of the form

$$
V(t, x(t))=x^{T}(t) P(t) x(t)
$$

By (i), we get

$$
\eta_{1}\|x(t)\|^{2} \leq V(t, x(t))=x^{T}(t) P(t) x(t) \leq \eta_{2}\|x(t)\|^{2} .
$$


The delta derivative of $V$ along the trajectories of system (2.8) is given by

$$
\begin{aligned}
V^{\Delta}(t)= & {\left[x^{T}(t) P(t)\right]^{\Delta} x(t)+x^{T}(\sigma(t)) P(\sigma(t)) x^{\Delta}(t) } \\
= & {\left[x^{T}(t) A^{T}(t)+f^{T}(t, x)\right] P(t) x(t)+\left[x^{T}(t)+\mu(t)\left(x^{T}(t) A^{T}(t)+f^{T}(t, x)\right)\right] } \\
& \times\left[P^{\Delta}(t) x(t)+P(t)(A(t) x(t)+f(t, x))+\mu(t) P^{\Delta}(t)(A(t) x(t)+f(t, x))\right] .
\end{aligned}
$$

By using (i), (ii), (iii), and Lemma 2.20, we obtain

$$
\begin{aligned}
V^{\Delta}(t, x(t)) & =\left[x^{T}(t) P(t) x(t)\right]^{\Delta} \leq \gamma_{1} \frac{h^{\Delta}(t)}{h(t)}\|x(t)\|^{2} \\
& \leq \begin{cases}\frac{\gamma_{1}}{\eta_{1}} \frac{h^{\Delta}(t)}{h(t)} V(t, x(t)), & h^{\Delta}(t) \geq 0, \\
\frac{\gamma_{1}}{\eta_{2}} \frac{h^{\Delta}(t)}{h(t)} V(t, x(t)), & h^{\Delta}(t)<0,\end{cases} \\
& \leq \frac{h^{\Delta}(t)}{h(t)}\left[x^{T}(t) P(t) x(t)\right] .
\end{aligned}
$$

From Gronwall's inequality for time scales [3], (i) and Lemma 2.15 in [2], we obtain

$$
\begin{aligned}
\eta_{1}\|x(t)\|^{2} & \leq x^{T}(t) P(t) x(t) \leq\left[x^{T}\left(t_{0}\right) P\left(t_{0}\right) x\left(t_{0}\right)\right] e_{h^{\Delta}(t) / h(t)}\left(t, t_{0}\right), \\
& \leq \eta_{2}\left\|x\left(t_{0}\right)\right\|^{2} e_{h^{\Delta}(t) / h(t)}\left(t, t_{0}\right) \leq \eta_{2}\left\|x\left(t_{0}\right)\right\|^{2} \frac{h(t)}{h\left(t_{0}\right)} .
\end{aligned}
$$

Hence, we get

$$
\|x(t)\| \leq \omega\left\|x\left(t_{0}\right)\right\| H(t) H\left(t_{0}\right)^{-1}, \quad t \geq t_{0},
$$

where $\omega=\sqrt{\eta_{2} / \eta_{1}}$ and $H(t)=\sqrt{h(t)}$. Therefore, the zero solution of (2.8) is $h$-stable.

Example 3.14. We consider the linear time-varying dynamic system of the form

$$
x^{\Delta}(t)=\left[\begin{array}{cc}
-a(t) & -1 \\
1 & -a(t)
\end{array}\right] x(t)+f(t, x(t)),
$$

where $a(t)=e_{\ominus 8}(t, 0)+1$ and $f(t, x(t))$ are rd-continuous in the first argument with $f(t, 0)=0$ for all $t \in \mathbb{T}$. Let $h(t)=5$ and

$$
f(t, x(t))=\left[\begin{array}{c}
0.125 \cos (t)\left[x_{2}(t)\right] \\
-0.125 \sin (t)\left[x_{1}(t)\right]
\end{array}\right] .
$$


Then $h^{\Delta}(t)=0$ and $\|f(t, x(t))\| \leq 0.125\|x(t)\|$. Let $\gamma=1 / 8, \epsilon_{1}=1, \epsilon_{2}=1 / 16, \epsilon_{3}=$ $1 / 2, \epsilon_{4}=1 / 16, \eta_{1}=1 / 8, \eta_{2}=1 / 4, \rho_{1}=-1$, and $\rho_{2}=0$. We can find a solution $P(t)$ satisfying (i)-(iii) of Theorem 3.13 as

$$
P(t)=\left[\begin{array}{cc}
\frac{1}{8} e_{\ominus 8}(t, 0)+\frac{1}{8} & 0 \\
0 & \frac{1}{8} e_{\ominus 8}(t, 0)+\frac{1}{8}
\end{array}\right] .
$$

Therefore, by Theorem 3.13, the system (3.39) is 5-stable.

\section{Conclusion}

In this paper, we have considered Lyapunov stability theory of linear time-varying system and derived sufficient conditions for uniform stability, uniform exponential stability, $\psi$ uniform stability and $h$-stability for linear time-varying system with nonlinear perturbation on time scales. By construction of appropriate Lyapunov functions, we have derived several stability conditions. Numerical examples are presented to illustrate the effectiveness of the theoretical results.

\section{Acknowledgments}

The first author is supported by Khon Kaen University Research Fund and the Development and the Promotion of Science and Technology Talents Project (DPST). The second author is supported by the Center of Excellence in Mathematics, CHE, Thailand. He also wish to thank the National Research University Project under Thailand's Office of the Higher Education Commission for financial support.

\section{References}

[1] S. Hilger, "Analysis on measure chains-a unified approach to continuous and discrete calculus," Results in Mathematics, vol. 18, no. 1-2, pp. 18-56, 1990.

[2] M. Bohner and A. Peterson, Dynamic Equations on Time Scales, Birkhäuser, Boston, Mass, USA, 2001.

[3] M. Bohner and A. Peterson, Advances in Dynamic Equations on Time Scales, Birkhäuser, Boston, Mass, USA, 2003.

[4] S. K. Choi, N. J. Koo, and D. M. Im, "h—stability for linear dynamic equations on time scales," Journal of Mathematical Analysis and Applications, vol. 324, no. 1, pp. 707-720, 2006.

[5] J. J. DaCunha, "Stability for time varying linear dynamic systems on time scales," Journal of Computational and Applied Mathematics, vol. 176, no. 2, pp. 381-410, 2005.

[6] I. B. Yaşar and A. Tuna, " $\psi$-uniformly stability for time varying linear dynamic systems on time scales," International Mathematical Forum, vol. 2, no. 17-20, pp. 963-972, 2007.

[7] S. Boyd, L. El Ghaoui, Eric Feron, and V. Balakrishnan, Linear Matrix Inequalities in System and Control Theory, vol. 15 of SIAM Studies in Applied Mathematics, Society for Industrial and Applied Mathematics (SIAM), Philadelphia, Pa, USA, 1994. 


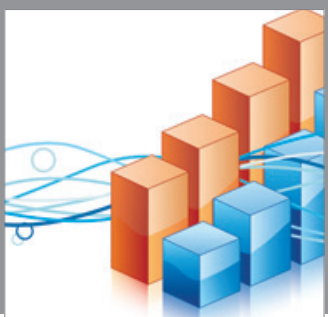

Advances in

Operations Research

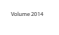

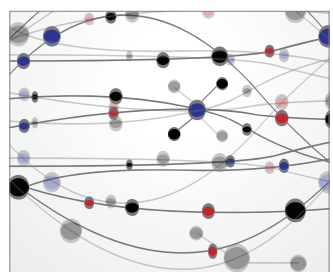

\section{The Scientific} World Journal
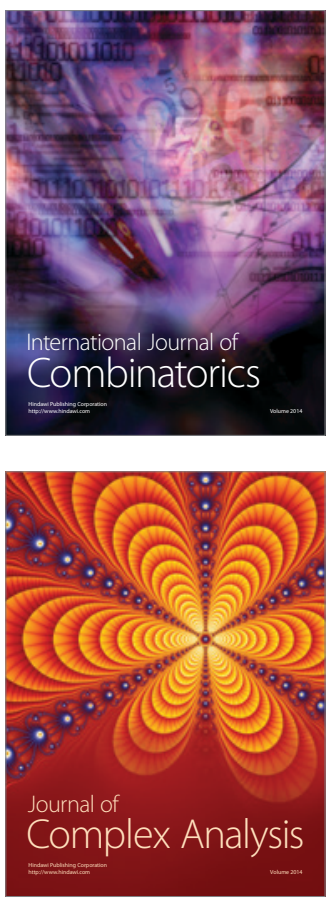

International Journal of

Mathematics and

Mathematical

Sciences
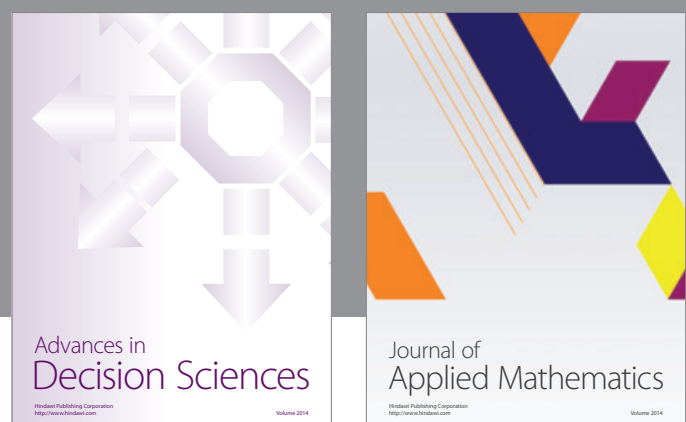

Journal of

Applied Mathematics
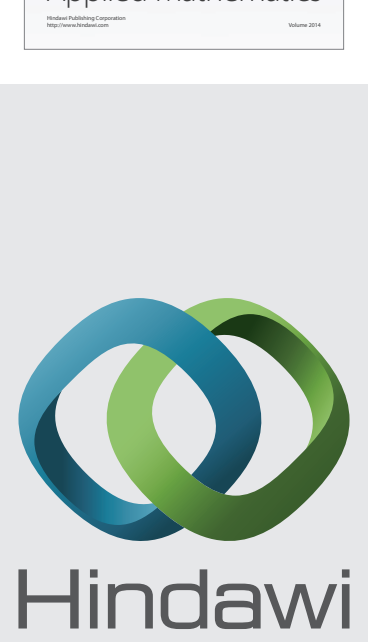

Submit your manuscripts at http://www.hindawi.com
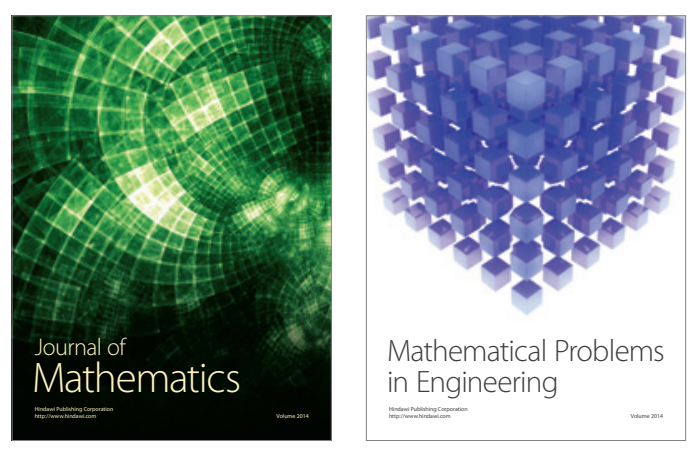

Mathematical Problems in Engineering
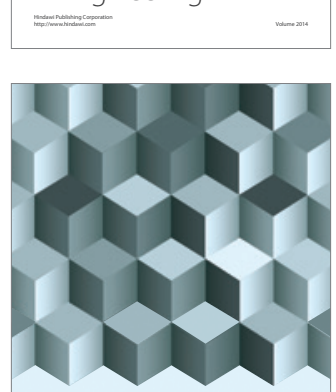

Journal of

Function Spaces
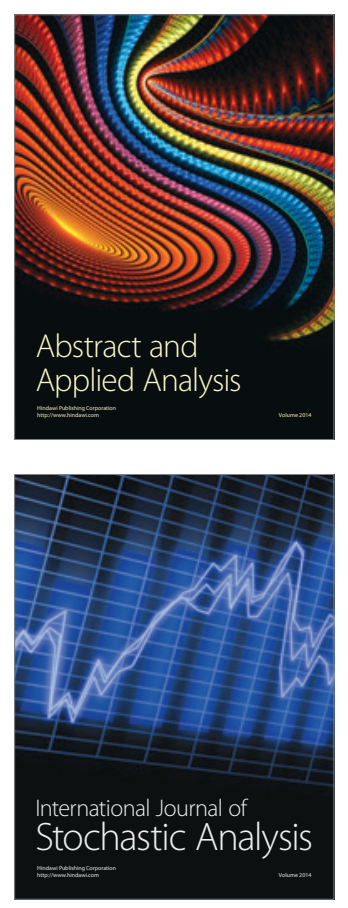

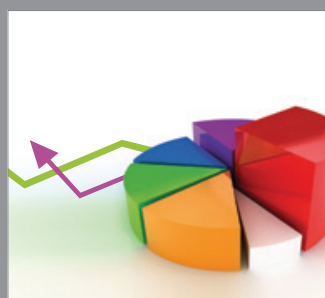

ournal of

Probability and Statistics

Promensencen
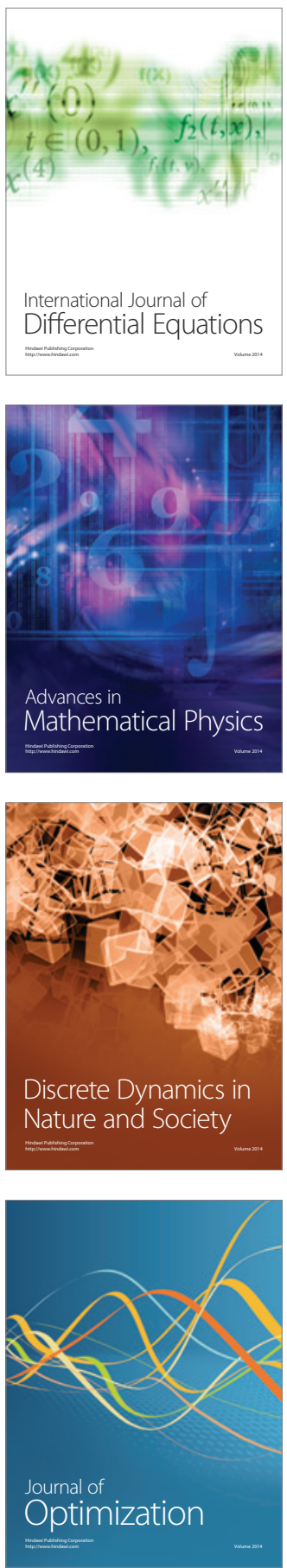